\title{
ASSESSMENT OF DRUG-RELATED PROBLEMS IN A TERTIARY CARE TEACHING HOSPITAL, INDIA
}

\section{SARFARAZ MOHAMMED*, SANJAY POUDEL, FERNANCE LALOO, ARJUN MADHUR, RINSON ROBERT, BINU MATHEW}

Department of Pharmacy Practice, NET Pharmacy College, Raichur, Karnataka, India. Email: sarfindia@gmail.com

\author{
Received: 14 October 2016, Revised and Accepted: 27 October 2016
}

\begin{abstract}
Objective: Drug-related problems (DRPs) are frequent in hospitalization where multiple changes in patient's medication regimen, and lack of continuity of care may be accompanied. The aim of present research was to identify DRPs, drug classes involved in DRPs as well as associated factors with the occurrence of DRPs and to assess the pharmacist interventions in a tertiary care teaching hospital.
\end{abstract}

Methods: A prospective observational study was carried out in a tertiary care teaching hospital, over a period of 6-month from November 2015 to April 2016. All the in patients admitted to all departments of hospital, who satisfied the selection criteria, were included in this study. Necessary demographic and clinical data were collected from the case records. The Pharmaceutical Care Network Europe Classification Version 5.01 was used to classify DRPs. The treatment data were analyzed to determine the rate, pattern, clinical significance, and outcomes of DRPs.

Results: A total of 300 patient case sheets were reviewed during the study, out of which 143 DRPs were identified from 93 patients. Male (\%) predominance was noted over females (\%). The most common DRP was drug interactions 47.55\% (68) followed by drug use problems 19.58\% (28), drug choice problems $14.68 \%$ (21), others $11.88 \%$ (17), dosing problems $4.89 \%$ (7), and adverse reaction $1.39 \%$ (2) were identified.

Conclusion: DRPs are common among the wards of hospital. Clinical pharmacist's role in identification, resolution, and prevention of DRPs helps in achieving better therapeutic outcomes and improved patient healthcare.

Keywords: Drug-related problems, Adverse drug reactions, Drug interactions, Drug choice problems, Dosing problem, Drug use problems, Pediatric, Medicine, Pharmaceutical Care Network Europe Classification.

(C) 2017 The Authors. Published by Innovare Academic Sciences Pvt Ltd. This is an open access article under the CC BY license (http://creativecommons. org/licenses/by/4. 0/) DOI: http://dx.doi.org/10.22159/ajpcr.2017.v10i2.15678

\section{INTRODUCTION}

Drug-related problems (DRPs) are events or circumstances involving drug therapy that actually or potentially interfere with desired health outcomes [1]. DRP can be manifested in several ways. DRPs may arise at all stages of the medication process from prescription to follow-up of treatment. Most of the problem usually occurs on administration, dispensing and during the patient's use of a medicinal product, but the lack of proper follow-up and reassessment of medical treatment by the physician is also a major problem [2]. DRPs occur more frequently in hospitalized patients where multiple changes are being made in patient's medication regimens, and lack of continuity of care may be accompanied [3]. The most common problems associated with drug use are many and includes inappropriate medication prescribing, discrepancies between prescribed and actual regimens, poor adherence, drug interactions (DI), inappropriate use, patients monitoring, inadequate surveillance for adverse effects, etc. DRPs lead to substantial morbidity, mortality as well as increased health-care expenditure which in turn affect the patient's quality of life [4]. A mean of categorizing DRPs is considered necessary to better focus the role of the pharmacist on the patient need and patient outcome [5]. Keeping in account, their characteristics and distinctions, all medicine-related problems can be classified in many ways. Furthermore, classification of DRPs can serve as a cynosure for establishing a systematic process for pharmacists to put in appreciably to positive patient outcomes [6]. Among classifications which are continuously tested is Pharmaceutical Care Network Europe Classification (PCNE) classification. Its basic classification has 4 primary domains for problems, 8 primary domains for causes, 5 primary domains for interventions, and 4 primary domains for outcome of intervention [7]. The role of pharmacists is expanding in primary care. Pharmaceutical care identifies and resolves actual or potential DRPs [8]. The traditional relationship between the doctor as prescriber, and pharmacist as dispenser, is no longer appropriate to ensure safety, effectiveness, and adherence to therapy. Pharmacists need to pay more attention to patient-centered, outcomes-focused care to optimize the safe and effective use of medicines [9]. Several studies conducted in developed countries shows the high evidence of drugrelated errors among hospitalized patients. The studies related to the prevalence of DRPs is lacking in India, however, some of the studies conducted Indian hospital shown much evidence of DRPs and also reflects positive impact of pharmacist intervention on reducing drugrelated errors and overall patients care [10]. Hence, the purpose of this study was to assess and evaluate the DRPs in a tertiary care teaching hospital.

\section{METHODS}

The prospective, observational, and interventional study was conducted for 6-month from November 2015 to April 2016 at Navodaya Medical College Hospital and Research Centre (NMCH \& RC), Raichur, Karnataka which is 1000 bedded multi-specialty tertiary care teaching hospital. Case sheets of patients who were admitted to all departments of the hospital and any disease and age group was taken for the study. The study was approved by Institutional Ethical Committee of the hospital and verbal consent from patients was taken. Exclusion criteria involved all the casebooks of outpatients. The study materials included the patient data entry form (includes patient and administration details), DRPs documenting form and PCNE guidelines. 
Pharmacist routinely monitored the patient's drug therapy during their hospital stay. The prescriptions were chosen randomly, and/or the details were followed till discharge of the patients. The data for the present study was collected by chart review method. During the study inpatient case, records/sheets were reviewed, which included patients case history, diagnosis, physician medication order sheets, nurse medication administration records, progress chart, laboratory investigations, and report of other diagnostic tests. The necessary information was documented in the patient profile form. Demographic data were calculated and expressed as percentages. All the case sheets were checked for DRPs by the Micromedex (mobile version), drugs. Com database and various textbooks along with the interview from patients and other health-care professionals and evaluated as per PCNE classification. The data from case sheets were checked for side effects both allergic and non-allergic, toxic effect, inappropriate drug or drug form, duplication of active ingredient, contraindication, treatment without indication, untreated indication, low dose and frequency, high dose and frequency, improper drug use, interaction, patient satisfaction, and other unclear complaints with therapy failure. Whenever DRP was identified, during the review, data from patient data entry form were transferred to DRPs documenting form.

The study data were analyzed using suitable statistical procedures in Microsoft Excel and word 2007. For descriptive statistics, results were expressed in terms of percentages and presented using tables and diagrams according to the types of tool used.

\section{RESULTS}

In the present study, an attempt was made to assess the DRP's in our NMCH \& RC - A tertiary care teaching hospital. The DRP's were assessed by identifying the problems, categorizing them which helps in designing the proper intervention based on the problems. The patient's data were collected as per standard data entry form and initially demographic analysis of the patients was done. The study showed that more male patients $(61.33 \%)$ were admitted to the hospital than female. It was observed that majority of the patients were unmarried $(52.67 \%)$. In this study, more number of patients enrolled was in the age group, 0-14 years (31\%). The demographic details are presented in Table 1 . The hospitalized inpatients were evaluated for various clinical characteristics. It was observed that ward wise distribution of the population showed more number of patients (25.33\%) admitted in general medicine ward. Out of 300 patients, majority patients were hospitalized for more than 5 days (46.67\%), which was followed by the patients hospitalized for 3-5 days (30.67\%) and patients hospitalized for $<3$ days (22.67\%). In this study, more number of the patients were free from any DRPs issues (69\%) (Table 2). This result may be due to the proper precaution by health-care professionals and patient during drug prescribing and drug use. In a total of 300 cases followed, 93 cases were identified with 143 DRPs. Among that 53.76\% (50) cases had only one problem, $38.70 \%$ (36) cases had two problems and remaining $7.52 \%$ (7) had three problems during their hospital stay. The overall incidence of DRPs was found to be $47.66 \%$. The clinical details of study subjects are given in Table 2 .

In the 143 DRPs identified, $1.39 \%$ (2) are adverse reactions, $14.68 \%$ (21) are drug choice problems, $4.89 \%$ (7) are dosing problem, $19.58 \%$ (28) are drug use problems, $47.55 \%$ (68) are drug interaction, and $11.88 \%$ (17) are other problems such as patient dissatisfaction, insufficient awareness of health and diseases, and therapy failure (Table 3). Age-wise distribution of DRPs showed a higher incidence of DRPs in the age group 25-39 years (Fig. 1).

When we come to domain classification of DRP's off the total 143 DRPs $1.39 \%$ (2) DRPs were side effect suffered (allergic) which comes under problem-adverse reaction, 6.30\% (9) DRPs were no drug prescribed for clear indication which comes under-drug choice problem, 2.09\% (3) DRPs were treatment duration too short which comes under dosing problem, $16.08 \%$ (23) DRPs were drug not taken at all which comes under problem drug use, $2.09 \%$ (3) DRPs were treatment duration too short which comes under dosing problem, 46.10\% (66) DRPs were potential interactions which come under problem drug interaction and 7.76\% (11) DRPs were insufficient awareness of health and disease which comes under other problems. The domain classification of DRPs according to PCNE classification (Version 5.01) is given in Table 4 . Class-wise categorization of drugs involved in DRP's indicated that the most problem causing drug was antibiotics (33.5\%) followed by drugs acting on gastrointestinal tract (GIT) $-29.3 \%$ as shown in Fig. 2 .

\section{DISCUSSION}

Drugs are the absolute therapeutic tools used in diseased condition in hospitalized patients. Drugs are intended to cure, prevent or diagnose

Table 1: Demographic details of study subjects $(n=300)$

\begin{tabular}{ll}
\hline Demographic variables & Inpatients \\
\cline { 2 - 2 } & Number of subjects (\%) \\
\hline Gender & $184(61.33)$ \\
$\quad$ Male & $116(38.67)$ \\
Female & \\
Marital status & $142(47.33)$ \\
Married & $158(52.67)$ \\
Unmarried & \\
Age in years & $93(31)$ \\
$0-14$ & $58(19.33)$ \\
15-24 & $62(20.67)$ \\
$25-39$ & $43(14.33)$ \\
$40-64$ & $44(14.66)$ \\
$>65$ & \\
\hline
\end{tabular}

Table 2: Clinical characteristics of study subjects $(n=300)$

\begin{tabular}{ll}
\hline Variables & Inpatients (n=300* \\
\cline { 2 - 2 } & Number of subjects (\%) \\
\hline Ward-wise distribution & $37(12.33)$ \\
Surgery & $33(11)$ \\
ICU & $71(23.66)$ \\
Pediatric & $76(25.33)$ \\
Medicine & $45(15)$ \\
Ortho & $38(12.66)$ \\
OBG & $68(22.67)$ \\
Hospital stay (no of days) & $92(30.67)$ \\
$<3$ & $140(46.67)$ \\
$3-5$ & \\
$>5$ & $93(31)$ \\
DRPs & $207(69)$ \\
Yes & No of DRP's in each case \\
No & $n=93$ ( $(\%)$ \\
No of DRP cases & $3(7.52)$ \\
& $2(38.70)$ \\
7 & $1(53.76)$ \\
36 & Total DRP's=143 in 93 cases \\
50 & \\
&
\end{tabular}

$*(n=300)$ represents total no of subjects $(n=93),{ }^{\prime}$ Represents total no of DRP cases. ICU: Intensive care unit, OBG: Obstetrics and Gynecology, DRP's: Drug-related problems

Table 3: Types of DRP's identified ( $n=143)$

\begin{tabular}{ll}
\hline Types of DRP's & Number of DRP's observed (\%) \\
\hline Adverse reaction & $2(1.39)$ \\
Drug choice problems & $21(14.68)$ \\
Dosing problems & $7(4.89)$ \\
Drug use problems & $28(19.58)$ \\
Interactions & $68(47.55)$ \\
Others & $17(11.88)$ \\
\hline
\end{tabular}

DRP's: Drug-related problems 
a disease, but improper use of drugs may cause patient morbidity and even mortality. Over the years, advancement in drug therapy has enhanced patient care but also led to increase in the incidence of DRPs. DRPs arise at all stages of the medication process from prescription to follow-up of the treatment. Clinical pharmacists are the heart of the clinical pharmacy services whose aim is safe and effective use of medicines. Clinical pharmacists play an important role in identifying DRP's, resolving actual DRP's, and preventing potential DRP's thereby improving the well-being of the patients.

In the present study, an attempt was made to assess the DRP's in NMCH \& RC a tertiary care teaching hospital. The DRP's were assessed by identifying the problems, categorizing them which helps in designing the proper intervention based on the problems. The patient's data were collected as per standard data entry form and initially demographic analysis of the patients was done. The study

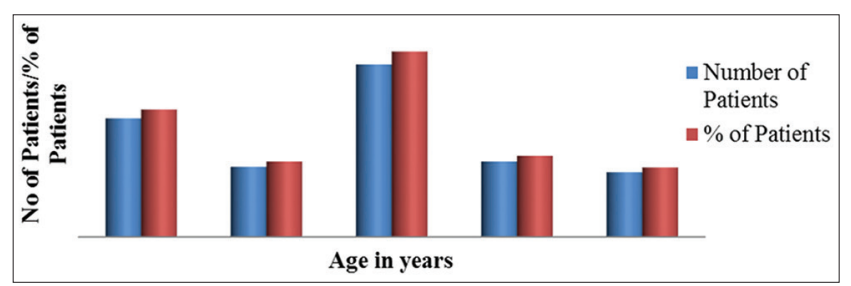

Fig. 1: Age-wise distribution of drug-related problems (DRPs) $(n=93) . n=93$ is number of subjects detected with DRP's

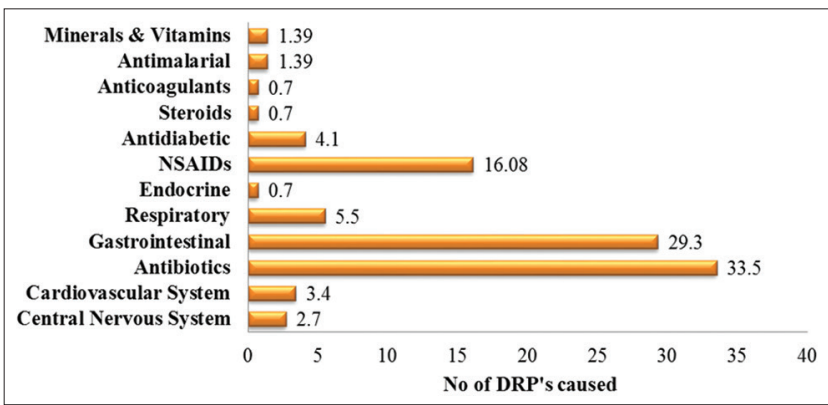

Fig. 2: Class-wise categorization of drugs involved in drug-related problems $(n=143)$ showed that more male patients were admitted to the hospital than female. A similar prospective observational and interventional study conducted by Ganachari et al., [11] from Belgaum, India also reported male $(58.06 \%)$ predominance over females (41.93\%). Furthermore, a similar observation was reported from Rijo et al., from Kochi, Kerala where $59.4 \%$ of male patients were admitted compared to $40.6 \%$ females [12]. It was observed that majority of the patients were unmarried (Table 1). More number of patients enrolled for the study was in age group, 0-14 years. This may be due to less admission of elderly patients because of the unpleasant ambulatory condition. The ward-wise distribution of the population showed more number of patients admitted in general medicine ward (Table 2). The geographical location, challenging climatic condition, and pollution of this place leading to seasonal diseases, infections, and allergies might be the cause for the higher admission of patients in medicine ward than others.

Of 300 patients, majority patients were hospitalized for more than 5 days, which is followed by the patients hospitalized for 3-5 days and patients hospitalized for $<3$ days (Table 2). It was observed that most of the patients visit the hospital only at the severe form of the simple disease. Thus the condition getting worst led them for prolonged hospital stay that will impact on their economic status. These can be avoided with proper awareness at the village and community level, more interactive health camps, posts and health education. It was observed that more number of patients were free from any DRPs issues (Table 2). In addition, a similar result was found in the study conducted by Ahmad and Khan [13]. This result may be due to the proper precaution by health-care professionals and patient during drug prescribing and drug use.

In total, 143 DRPs were identified in 93 patients. Although the patients with DRPs are less, the total number of DRPs is more which is due to the presence of more than one DRP in one case of the patient. Of the 93 patients, the majority of them had 1 DRPs followed by patients with 2 DRPs and patients with 3 DRPs (Table 2). The contributing factors are perhaps polypharmacy, prescribing multiple drugs for the same patient leading to more than one DRP. This report is similar to one reported by Arvind and Shobha [14]. The gender wise distribution of DRPs study showed higher incidence of DRPs in males $58.06 \%$ over females $41.94 \%$. A similar result was observed with study conducted by Dinesh et al., [15]. This may be attributed to the fact that more number of patient cases selected for study was males than females. Age-wise distribution of DRPs showed a higher incidence of DRPs in

Table 4: Domain classification of DRPs $(n=143)$

\begin{tabular}{|c|c|c|}
\hline Code & Problems & Number of problems $(\%)$ \\
\hline \multirow[t]{2}{*}{ P1 } & Adverse reaction & \\
\hline & Side effects suffered (allergic) & $2(1.39)$ \\
\hline \multirow[t]{5}{*}{$\mathrm{P} 2$} & Drug choice problems & \\
\hline & Inappropriate drug & $2(1.39)$ \\
\hline & Inappropriate duplication of therapeutic group or active ingredient & $5(3.50)$ \\
\hline & No clear indication for drug use & $5(3.50)$ \\
\hline & No drug prescribed but clear indication & $9(6.29)$ \\
\hline \multirow{3}{*}{ P3 } & Drug dose too low or dosage regimen not frequent enough & $2(1.39)$ \\
\hline & Drug dose too high or dosage regimen too frequent & $2(1.39)$ \\
\hline & Duration of treatment too short & $3(2.09)$ \\
\hline \multirow[t]{3}{*}{$\mathrm{P} 4$} & Drug use problems & \\
\hline & Drug not taken/administered at all & $23(16.08)$ \\
\hline & Wrong drug taken/administered & $5(3.50)$ \\
\hline \multirow[t]{2}{*}{ P5 } & Drug interaction & \\
\hline & Potential interaction & $66(46.1)$ \\
\hline \multirow[t]{4}{*}{ P6 } & Others & \\
\hline & Patient dissatisfied with therapy despite taking drug (s) correctly & $4(2.83)$ \\
\hline & Insufficient awareness of health and diseases & $11(7.66)$ \\
\hline & Therapy failure (reason unknown) & $2(1.39)$ \\
\hline
\end{tabular}


the age group 25-39 (34.40\%). This may be due to more no of drugs prescribed to this age group and the fact that second large number of patient cases selected for the study was also from this age group. Although the number of patients enrolled in the study was more from the age group 0-14 years, the problems associated with this age group was less. This may be because the proper care in drug use was provided in the pediatrics than the other age group. The administration of drugs in pediatric patients was guarded by parents, which also results in reduction in DRPs. When it comes to domain classification of DRP's, the most problem causing drug was antibiotics (33.5\%) followed by drugs acting on GIT (29.3\%). It might be due to the reason that the antibiotics are prescribed more and used irrationally. The unavailability of therapeutic drug monitoring might also be the associated reason for antibiotics causing problems (Fig. 2). In the study, the least DRP was an adverse reaction (allergic) which is seen in only 2 patients. The drug associated with the reaction was antibiotics, and the reason was lack of hypersensitivity testing before the drug administration. The example for adverse reaction identified in this study was skin rashes due to gentamycin injection. Proper screening for any hypersensitivity of the antibiotics must be done before the administration of the drug to decrease this problem. Another DRP found was drug choice problem. The contributing factor was mainly improper prescribing by physician, improper nursing, and pharmacist supervision and the uncertain diagnosis leads to untreated indication. The drug associated was NSAIDs and drugs acting on GIT (ranitidine, etc.) followed by antibiotics. Lesser dosage and short duration of treatment were the most frequent DRP's in dosing problem. The main reason was patient noncompliance which is either due to improper counseling or patient attitude to treatment and the early removal of symptoms with an initial incomplete dose of drugs (antibiotics). Therefore, efforts should be made to minimize these dosing errors such as the involvement of a pharmacist in deciding the dosing of drugs or a computerized dosing program.

In DRP belonging to drug use problem, two problems (drugs not taken and wrong drug taken) were noticed. These problems occurred at the patient level, i.e., because of the patients themselves. The physician prescribes the medicine but due to patient's problems such as poverty, waiting of free distribution medicines, halting the medicines soon after the symptoms subsides and less knowledge regarding the drugs, these problems are arising. Mostly, the drug not taken was followed by wrong drug taken. Patients who are staying in wards for long time are buying the medicines for other patients of same ward also which leads to confusion and wrong drugs taken. Mostly, these problems are associated with the oral medicines where patients themselves administer the drugs. Some patients because of language barrier cannot interact with the health-care provider regarding the drug confusion ending up with wrong drug administration.

Drug interaction $(47.55 \%)$ was the predominantly occurring problem. The findings were similar to studies by Dasari et al., who carried out DRP studies in a South Indian hospital [16] and Rijo et al., who conducted the similar study in Kochi [12]. This may be because prescribed antibiotics, anticoagulants are the drugs that interact with large number of other drugs, lack of medication history, lack of physician knowledge about drugs and polypharmacy. Few examples of drug-DI identified in this study involve drug interaction between ranitidine and theophylline, digoxcin with calcium channel blocker, etc. The DI identified in this study were mostly based on established literature and evidence (mostly potential). In clinical practice, several drugs can still be used together, yet close monitoring is fundamental, and any toxicity should be identified and immediately followed by corrective actions. Many of the patients in this study did not engage in regular physical activity, did not adhere to proper diets, did not perform any routine blood monitoring, and defaulted on follow-up or medications. This leads to others problems like therapy failure and also a problem of insufficient awareness.

\section{CONCLUSION}

Drugs can be useful tools in the prevention and treatment of symptoms and diseases, but if not used properly, they may be harmful and cause new symptoms or produce sub-optimal effects which are termed as DRPs. Polypharmacy, comorbidities, lack of awareness has been found as the reason for DRPs. This study presents a pattern of finding DRPs in a private tertiary care teaching hospital in India. The present results point to the establishment of a DRPs reporting system at each hospital and to share the data with other hospitals/healthcare settings. The role of a clinical pharmacist in this situation appears to be a strong intervention and very crucial one which depends on the pharmaceutical services provided by them and, the clinical pharmacist, initially, could only confine to identification of the DRPs, later bring those problems to the notice of physician and other healthcare professional and take precaution and measures to avoid them. Our study shows that DRPs are significantly occurring in hospital causing the patient for comorbidity, prolonged hospitalization, and increased financial burden. The study suggests that the pharmacists and general practitioners (physicians) can work together to identify and resolve DRPs.

\section{REFERENCES}

1. Pharmaceutical Care Network Europe Homepage. Available from: http://www.pcne.org.

2. Viktil KK, Blix HS. The impact of clinical pharmacists on drugrelated problems and clinical outcomes. Basic Clin Pharmacol Toxicol 2008;102(3):275-80

3. Blix HS, Viktil KK, Reikvam A, Moger TA, Hjemaas BJ, Pretsch P, et al. The majority of hospitalised patients have drug-related problems: Results from a prospective study in general hospitals.Eur J Clin Pharmacol 2004;60(9):651-8.

4. Parthasarathi G, Ramesh M, Kumar JK, Madaki S. Assessment of drugrelated problems and clinical pharmacists' interventions in an Indian teaching hospital. J Pharm Pract Res 2003;33(4):272-4.

5. Strand LM, Morley PC, Cipolle RJ, Ramsey R, Lamsam GD. Drug-related problems: Their structure and function. DICP 1990;24(11):1093-7.

6. Ulrika G. Effects of clinical pharmacist's interventions on drug-related hospitalization and appropriateness of prescribing in elderly patients. Digitala Vetenskapliga Arkivet 2012;154:58.

7. Available from: http://www.pcne.org/working-groups/2/drug-relatedproblems.

8. Adusumilli PK, Adepu R. Drug related problems: An over view of various classification systems. Asian J Pharm Clin Res 2014;7(4):7-10.

9. Debbie R. Collaboration between doctors and pharmacists in the community. Aust Prescr 2010;33(6):191-3.

10. Kumar BP, Prasanna D, Rajesh V, Prashant CF. Assessment of clinical pharmacist intervention in tertiary care teaching hospital of southern India. Asian J Pharm Clin Res 2013;6(2):258-61

11. Ganachari MS, Kumar M, Shashikala C, Fibin M. Assessment of drug therapy interventions by clinical pharmacist in a tertiary care hospital. Indian J Pharm Pract 2010;3(3):22-8.

12. Rijo M, Emmanuel J, Vijayalakshmi S. Clinical pharmacist's interventions on drug related problems in a tertiary care hospital. Int $\mathrm{J}$ Pharm Pharm Sci 2015;7(6):401-4

13. Ahmad A, Khan MU. The impact of clinical pharmacist interventions on drug related problems in a teaching based hospital. IJPCR 2014;6(2):276-80

14. Arvind K, Shobha C. Assessment of drug-drug interactions in hospitalizes patients in India. Asian J Pharm Clin Res 2011;1(4):62-5.

15. Dinesh R, Ramani JU, Nagar AK, Yogananda M, Bharathi DR. Identification and evaluation of drug related problems in medicine ward at a tertiary care teaching hospital. Am J Pharm Health Res 2014;2(11):182-94.

16. Dasari JR, Banothu VP, Vemulapally R, Sangram V. Identification and assessment of drug related problems in stroke patients admitted to a tertiary care hospital. Res J Pharm Biol Chem Sci 2016;7(4):1607-15 